\title{
Comment on Pregnancy-Induced Hemophagocytic Syndrome
}

\author{
Koduri Prasad R.
}

Received: 5 September 2013/ Accepted: 8 May 2014/Published online: 24 May 2014

(C) Federation of Obstetric \& Gynecological Societies of India 2014

\section{To the Editor,}

I read with much interest the paper by Shukla et al. that appeared in a recent issue of the Journal (2013;63:203205). The diagnosis of hemophagocytic syndrome can be considered established in the patient in light of the prolonged fever, pancytopenia, hyperferritinemia, hypertriglyceridemia, and splenomegaly.

It needs mention that the microscopic visualization of hemophagocytosis is simply one of the possible diagnostic criteria and does not necessarily need to be present to establish the diagnosis of hemophagocytic lymphohistiocytosis [1].

The performance of liver biopsy in the pregnant woman with thrombocytopenia as described by the authors may have been unnecessary. Liver, spleen, and lymph node biopsies merely for demonstration of hemophagocytosis should be avoided [2].
Could the patient have had an undiagnosed infection by an obligate intracellular microbe e.g., Salmonella typhi or Brucella sp. that could have triggered the hemophagocytic syndrome? Details of antimicrobial therapy and the number of antibiotic days prior to the patient having the spontaneous abortion with resolution of the hemophagocytic syndrome are, thus, of interest.

\section{References}

1. Rosado FGN, Kim AS. Hemophagocytic lymphohistiocytosis. An update on diagnosis and pathogenesis. Am J Clin Pathol. 2013;139:713-27.

2. Lehmberg K, Stephan E. Diagnostic evaluation of patients with suspected haemophagocytic lymphohistiocytosis. Br J Haematol. 2013;160:275-87.

Koduri P. R.

Division of Hematology-Oncology, The Department of

Medicine, Mediciti Hospital, Hyderabad 500 029, India

Koduri P. R. ( $\square)$

Mediciti Hospital, Secretariat Road, Hyderabad 500063, AP., India

e-mail: prkoduri@yahoo.com 